\title{
Detection of a large amount of diffuse extraplanar dust in NGC 891
}

\author{
Kwang-Il Seon ${ }^{1}$ and Adolf N. Witt ${ }^{2}$ \\ ${ }^{1}$ Korea Astronomy and Space Science Institute, Republic of Korea \\ email: kiseon@kasi.re.kr \\ ${ }^{2}$ Ritter Astrophysical Research Center, University of Toledo, USA \\ email: awitt@utnet.utoledo.edu
}

\begin{abstract}
Significant discrepancies have been found between the dust masses derived from various tracers (optical/near-IR, far-IR/sub-millimeter observations, and the variation of dust attenuation with viewing angle). Here we report the first detection of the extended far-UV (FUV) and near-UV (NUV) haloes perpendicular to the galactic plane of NGC 891, which can be interpreted as scattered stellar light from the galactic plane. An additional "geometrically thick" dust disk, which contains about the same mass as the standard thin dust disk, is needed to reproduce the vertically extended UV profiles
\end{abstract}

Keywords. radiative transfer, galaxies: spiral, galaxies: individual (NGC 891)

\section{Energy balance problem in spiral galaxies}

Internal dust attenuation of the photon flux from galaxies reduces the total emergent luminosity of galaxies, introducing an inclination dependence of the observed flux. The amount of dust may then be inferred from the inclination dependence of the dust attenuation. The classical test to examine the opacity of spirals, dust attenuation versus the inclination angle, however, caused debates over whether face-on spiral galaxies are optically thin or thick (Valentijn 1990; Burnstein et al. 1991). Motivated by these arguments, realistic radiative transfer models for dusty galaxies were developed and compared with optical/near-IR (NIR) images (e.g., Kylafis \& Bahcall 1987; Xilouris et al. 1997; Xilouris et al. 1998). The result seemed to confirm the conventional viewpoint that spiral galaxies are optically thin. However, comparisons between the optical/NIR and far-IR (FIR)/submm observations have been puzzling. The SED in FIR/submm requires at least a dust mass twice as large as estimated from the radiative transfer model of optical/NIR images (Popescu et al. 2000; Bianchi 2008). To resolve this discrepancy, an extra dust mass in the form of a secondary thin (even thinner than the thin disk found from optical/NIR observations) disk + clumpy dust clouds associated with molecular clouds that was supposed to be hidden in optical images was introduced (Popescu et al. 2000; Tuffs et al. 2004; Bianchi 2008; Popescu et al. 2011). However, the model produces a dust lane in K-band that is much stronger than that observed (Dasyara et al. 2005; Bianchi 2008).

We note that the previous radiative models assumed that the geometrically thin dust disk and/or the dust clouds are concentrated in the galactic midplane. We may thus infer that the conventional thin-disk picture of dust may have a limitation in explaining the observational facts consistently. In other words, an additional dust component that is existing in a form different from the standard thin disk and containing a significant amount of mass may be needed to overcome the discrepancies. 
In fact, there have been various attempts to determine whether large amounts of dust reside outside the galactic plane. In addition, the possibility of dust being repelled away from the galactic plane by radiation pressure of starlight in the disk has also been theoretically investigated (Greenberg et al. 1987; Ferrara et al. 1990). Filamentary dust structures above the galactic plane have been observed up to $|z| \lesssim 2 \mathrm{kpc}$ in nearby edge-on spiral galaxies using high-resolution optical images (e.g., Howk \& Savage 1997; Thompson et al. 2004). The extraplanar dust filaments were found to contain too small an amount of dust $(\sim 1-2 \%$ of the total dust mass) to be considered as the additional dust component (Popescu et al. 2000). The filamentary features, however, were traced in absorption against the background starlight, thereby implying preferentially "dense" dust features visible only to heights limited by the vertical extent of the background starlight. Therefore, any "diffuse" dust component above the galactic plane was not traceable in the studies and the full vertical extent and overall amount of the diffuse extraplanar dust remained unknown.

\section{Search for the vertically extended dust layer}

If there is a significant amount of diffuse dust above the galactic plane, the extraplanar dust may provide some, if not all, of the extra dust mass required to explain FIR/submm observations. This possibility was investigated by searching the vertically extended submm (450 and $850 \mu \mathrm{m}$ ) emissions in SCUBA images of NGC 891 (Alton et al. 2000a; Alton et al. 2000b). However, detection of a significant amount of extraplanar dust was not successful because the instrumental point spread function (PSF) was too broad, made worse by the presence of a wide wing (Alton et al. 2000a). Recently, Bianchi \& Xilouris (2011) analyzed Herschel/SPIRE images of NGC 891 at 250, 350, and $500 \mu \mathrm{m}$ and found no excess emission above the galactic plane beyond that of the thin, unresolved, disk. However, we should note that the spatial resolution of Herschel/SPIRE $\left(18.1^{\prime \prime}, 24.9^{\prime \prime}\right.$, and $36.4^{\prime \prime}$ FWHM for 250, 350, and $500 \mu \mathrm{m}$, respectively) is even worse than that of SCUBA (16" FWHM).

Here we report the results of a search for the diffuse extraplanar dust based on the fact that the dust should appear as a faint extended reflection nebula illuminated by starlight. The scattered light would not be easily distinguished from direct starlight when the scaleheight of the light source is greater than or comparable to that of the extraplanar dust. Therefore, UV observations of edge-on galaxies can provide the means for detecting scattered light from the diffuse extraplanar dust, because OB stars, the main source of the UV continuum, have a scaleheight $<0.2 \mathrm{kpc}$ and have no bulge or halo component. We thus examined the FUV data of the edge-on galaxy NGC 891 obtained from the GALEX mission (Gil de Paz et al. 2007), and indeed found vertically extended FUV and NUV emission in the galaxy.

Radiative transfer models of the dust-scattered UV radiation were calculated using a Monte-Carlo simulation code (Seon 2009). We assumed that stars and dust are exponentially distributed in both vertical and radial directions. The dust scattering properties were assumed to be identical to those of Milky Way dust. The models with a single exponential dust disk were obviously not able to produce the vertical profile of the data. Two exponential (geometrically thin + thick) dust disks were needed to reproduce the UV data (Fig. 1). The result indicates that an extraplanar dust disk with a mass as large as the standard thin dust disk, but with a 10 times higher scaleheight, is required to explain the UV profiles.

We note that the thick disk exhibits very little concentration toward the central plane compared to the thin dust disk. The vertical opacity profile of the two dust disks is in 

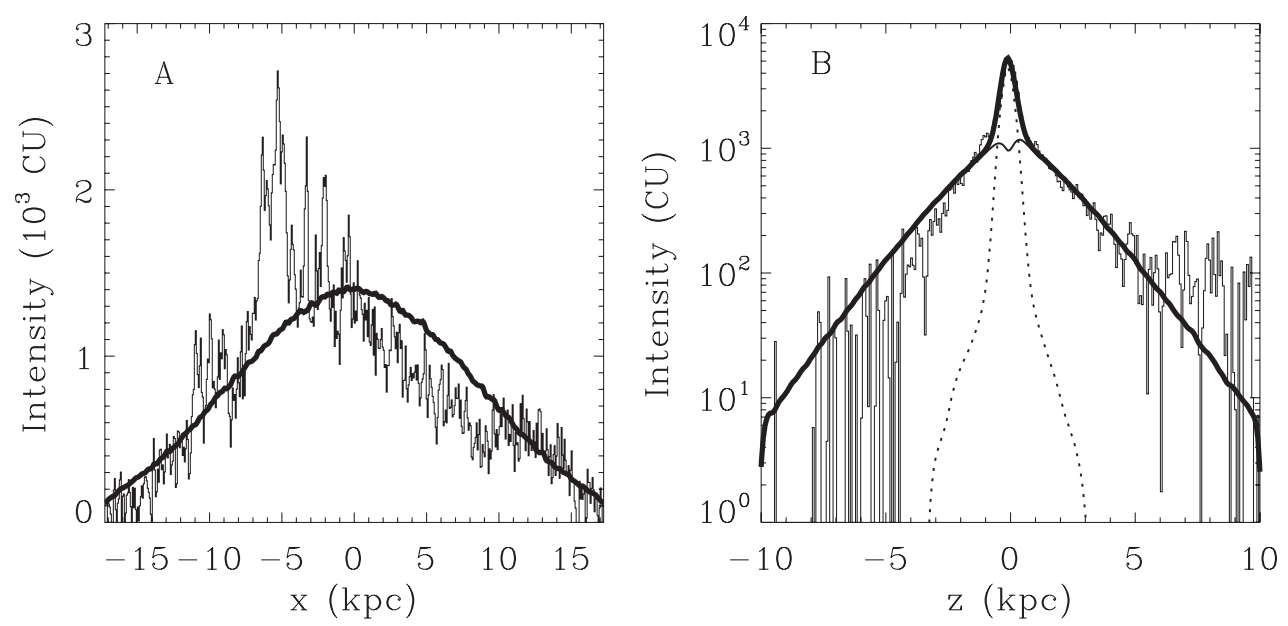

Figure 1. Observed FUV intensity profiles of NGC 891 plotted together with the corresponding profiles from the radiative transfer model (smooth thick solid line). (A) The radial intensity profile and (B) the vertical intensity profile. Smooth thin dotted and solid lines in (B) are the model profiles of directly escaped and scattered radiations, respectively. The broad wing features of the direct starlight in (B) are due to the Galex PSF. Obviously, the effect of the wing of the PSF is negligible. Here, 1 continuum unit $(\mathrm{CU})=1$ photons $\mathrm{cm}^{-2} \mathrm{~s}^{-1} \AA^{-1} \mathrm{sr}^{-1}$.

fact well represented by a single exponential with a scaleheight of $0.2 \mathrm{kpc}$, which are consistent with optical/NIR observations, in the central extinction-lane region $(|z|<$ $0.5 \mathrm{kpc}$ ) where models of optical/NIR images are mainly fitted. Therefore, the secondary thick disk can be completely hidden from the radiative transfer models of optical/NIR images when only a single dust disk is assumed.

\section{References}

Alton, P. B., et al. 2000, A\&SAS, 145, 83

Alton, P. B., et al. 2000, A\&A, 356, 795

Bianchi, S., Davies, J. I., \& Alton, P. B. 2000, $A \& A, 359,65$

Bianchi, S. 2008, A\&̈A, 490, 461

Bianchi, S. \& Xilouris, E. M. 2011, A\&A, 531, L11

Burnstein, D. Haynes, M., \& Faber, S. M. 1991, Nature, 353, 515

Davies, J. I., Phillipps, S., Boyce, P. J., \& Disney, M. J. 1993, MNRAS, 260, 491

Dasyra, K. M., et al. 2005, A\&GA, 437, 447

Ferrara, A., Ferrini, F., Barsella, B., \& Aiello, S. 1990, A\&\&A, 240, 259

Gil de Paz, A., et al. 2007, ApJS, 173, 185

Greenberg, J. M., Ferrini, F., Barsella, B., \& Aiello, S. 1982, Nature, 327, 214

Howk, J. C. \& Savage, B. D. 1997, AJ, 114, 2463

Kylafis, N. D. \& Bahcall, J. N. 1987, ApJ, 317, 637

Popescu, C. C., et al. 2000, A\&GA, 362, 138

Popescu, C. C., et al. 2011, A\&A, 527, A109

Seon, K.-I. 2009, ApJ, 703, 1159

Thompson, T. W. J., Howk, J. C., \& Savage, B. D. 2004, AJ, 128, 662

Tuffs, R. J., et al. 2004, A\&A, 419, 821

Valentijn, E. A. 1990, Nature, 346, 153

Xilouris, E. M., Kylafis, N. D., Papamastorakis, J., Paleologou, E. V., \& Haerendel, G. 1997, $A ध A, 325,135$

Xilouris, E. M., Alton, P. B., Davies, J. I., Kylafis, N. D., Papamastorakis, J., \& Trewhella, M. 1998, A\&A, 331, 894 